\title{
CINÉTICA DE SECADO DE Pisum sativum L. (ARVEJA VERDE) VARIEDAD USUI
}

\author{
Drying kinetics of Pisum sativum L. (Green peas) variety Usui \\ Javier S. Córdova ${ }^{1,2}$, José R. Juárez ${ }^{1}$, Luz M. Cerrón² \\ ${ }^{1}$ Facultad de Farmacia y Bioquímica, Universidad Nacional Mayor de San Marcos. ${ }^{2}$ Universidad Nacional del Centro del Perú

\section{RESUMEN} \\ La presente investigación tuvo como objetivo determinar los parámetros de cinética de secado de Pisum sativum L., \\ tales como: velocidad y tiempo de secado, humedad de equilibrio y crítica; los cuales son muy importantes para un secado \\ eficiente y de calidad. Además, se estudió la eliminación de agua presente en el alimento, para lo cual se utilizó un secador de \\ bandejas por aire caliente a $50^{\circ} \mathrm{C}$, determinándose el contenido de humedad del alimento a diferentes tiempos de exposición \\ a dicho aire, hasta lograr el peso constante del alimento. Los resultados obtenidos fueron: tiempo y velocidad de secado de \\ Pisum sativum L. (arveja verde), 594,36 minutos y o,00108 $\mathrm{gH}_{2} \mathrm{O} / \mathrm{m}^{2}$.min, respectivamente; humedad de equilibrio, o,071 \\ $\mathrm{gH}_{2} \mathrm{O} / \mathrm{g}$ sólidos secos, y humedad crítica, o, $5 \mathrm{O} 1 \mathrm{~g} \mathrm{H}_{2} \mathrm{O} / \mathrm{g}$ sólidos secos. Los valores mencionados fueron analizados mediante \\ la curva de secado y determinados por el método analítico de la teoría de secado de alimentos.
}

Palabras claves: Arveja verde, secado, velocidad de secado, tiempo, humedad, Pisum sativum L.

\section{SUMMARY}

The present study aimed to determine the drying kinetics parameters in Pisum sativum L., such as speed and drying time, equilibrium moisture and critical moisture; wich are very important for efficient drying and quality. Moreover, was studied removal of water present in the food, for which was used a tray dryer with hot air at $50{ }^{\circ} \mathrm{C}$, determining moisture content of the food at different times of exposure to hot air, until achieving constant weight of food. The results obtained were: drying time and speed of Pisum sativum L. (peas green), 594,36 minutes and o,oo108 $\mathrm{gH} \mathrm{H}_{2} \mathrm{O} / \mathrm{m}^{2} . \mathrm{min}$, respectively; equilibrium moisture, $0,071 \mathrm{gH}_{2} \mathrm{O} / \mathrm{g}$ dry solids, and critical moisture, o,501 $\mathrm{gH}_{2} \mathrm{O} / \mathrm{g}$ dry solids. The values obtained were analyzed using the drying curve and determined by the analytical method of food drying theory.

Keywords: Green peas, drying, drying speed, time, humidity, Pisum sativum L.

\section{INTRODUCCIÓN}

a arveja fresca se comercializa en los mercados de abasto, a precios diferentes que suelen ser rentables para los productores; en el caso de no lograse la venta en su estado fresco, una de las alternativas es secarla y venderla como producto seco. La deshidratación es una de las operaciones más antiguas usadas para conservar alimentos, dando lugar a productos secos tradicionales como carnes, pescados, frutas y quesos, entre otros ${ }^{(1)}$. En las últimas décadas, "nuevos" productos, llamados alimentos de humedad intermedia o baja, tales como café soluble, leche en polvo y las formulaciones deshidratadas para la preparación de puré de papa, han tenido un éxito notable, se trata de una operación unitaria utilizada en las diferentes industrias del procesamiento de alimentos a nivel artesanal e industrial.

\section{MATERIALES Y MÉTODOS}

El trabajo se desarrolló en el laboratorio de Análisis y Tecnología de Alimentos de la Facultad de Ingeniería en Industrias Alimentarias - Universidad Nacional del Centro del Perú. Se procedió a deshidratar Pisum sativum L. (arveja verde) variedad Usui, en un secador de cuatro (o4) bandejas, basado en el método de Geankoplis ${ }^{(2)}$.

\section{Determinación de los datos experimentales}

El equipo experimental (secador de bandejas), fue lo más semejante posible al secadero donde se efectúa realmente el secado de la arveja verde y las condiciones del aire fueron constantes. El trabajo consistió en registrar el cambio de masa del alimento en el tiempo.

\section{Determinación de humedades}

Se han determinado: humedad crítica, humedad de equilibrio y humedad libre, a diferentes tiempos de análisis y control.

\section{Determinación del tiempoyvelocidad de secado}

Con los datos experimentales se ha determinado analíticamente el tiempo y velocidad de secado y, a partir de esto, se elaboraron las curvas respectivas. 


\section{RESULTADOS}

La arveja verde se secó en un secador de bandejas, teniendo en cuenta que la bandeja y la muestra tuvieron un peso de 299,1 y 120,o g, respectivamente. Luego se realizaron los cálculos referentes al secado, a partir de los datos experimentales obtenidos durante dicho proceso, determinándose la curva de secado

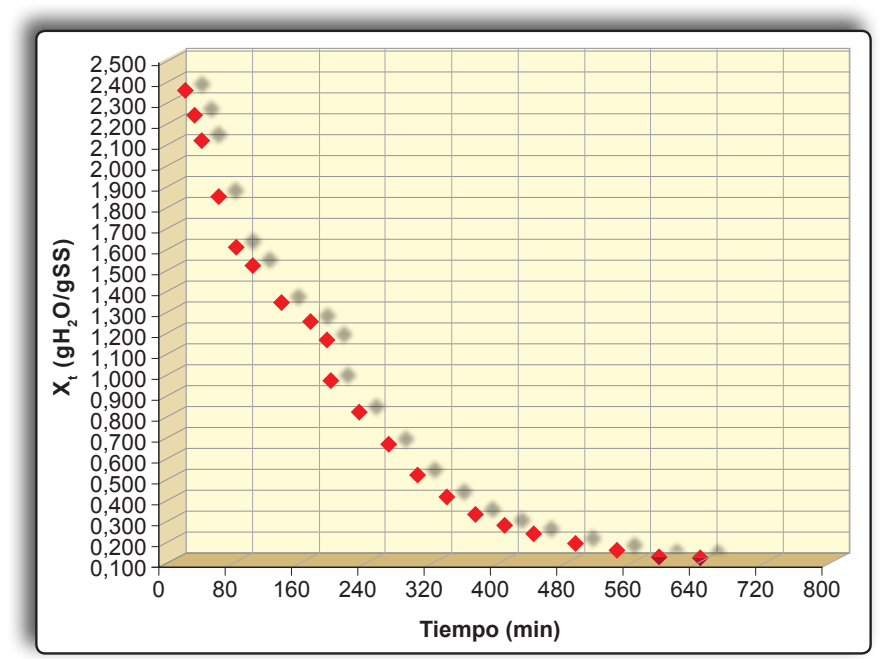

Figura 1. Curva de secado de la arveja: $X_{t}$ - Tiempo.

$\mathrm{X}_{\mathrm{t}}$ : Humedad libre total en $\mathrm{gH}_{2} \mathrm{O} / \mathrm{g}$ sólidos secos

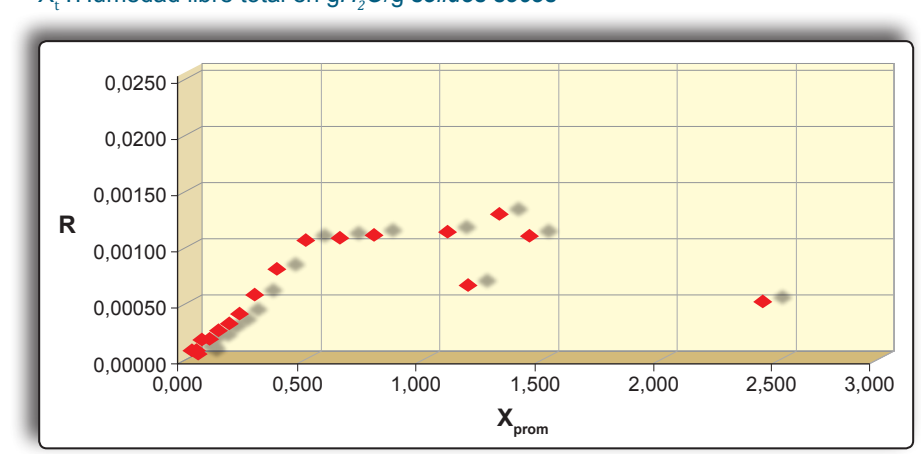

Figura 2. Curva de secado de la arveja: $\mathrm{R}-\mathrm{X}_{\text {prom}}$.

$\mathrm{X}_{\text {prom }}$ : Humedad libre promedio en $\mathrm{gH}_{2} \mathrm{O} / \mathrm{g}$ sólidos secos

$\mathrm{R}^{\text {prom }}$ : Velocidad de secado en $\mathrm{gH}_{2} \mathrm{O} / \mathrm{m}^{2}$. min

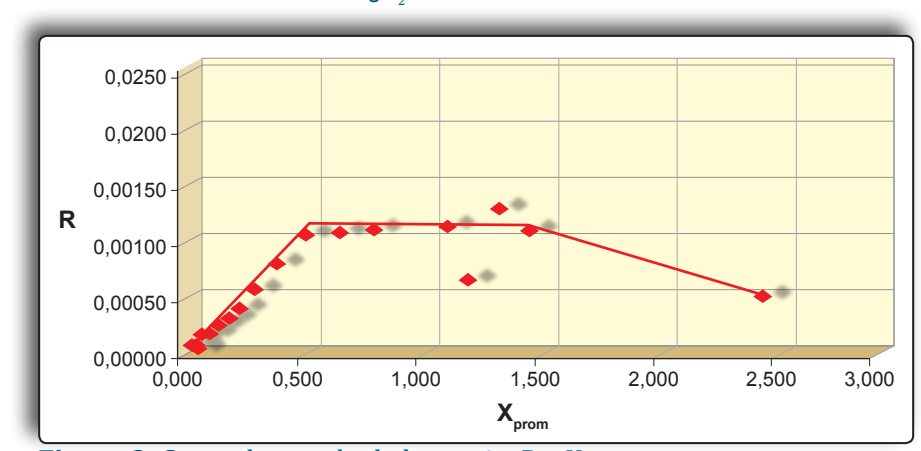

Figura 3. Curva de secado de la arveja: $\mathrm{R}-\mathrm{X}_{\text {prom }}$.

$\mathrm{X}_{\text {prom }}$ : Humedad libre promedio en $\mathrm{g}_{2} \mathrm{O} / \mathrm{g}$ sólidos secos

$\mathrm{R}^{\text {prom }}$ : Velocidad de secado en $\mathrm{gH}_{2} \mathrm{O} / \mathrm{m}^{2}$. min y otros parámetros que corresponden a la cinética (figuras 1, 2 y 3 ).

Se utilizaron métodos analíticos descritos en la teoría de secado de alimentos, para determinar la humedad de equilibrio y sólidos secos, siendo los resultados 0,071 $\mathrm{gH}_{2} \mathrm{O} / \mathrm{g}$ sólidos secos y 33,6 g, respectivamente. Respecto a la humedad crítica, se determinó de la misma forma, y con este valor se halló la velocidad de secado de la arveja (tabla 1).

En consecuencia, luego de obtener los resultados del secado de la arveja, se determinó la función o regla de correspondencia de la inversa de velocidad de secado en función de la humedad promedio, dada por:

$$
\begin{aligned}
& \mathrm{Y}=706,3 \mathrm{X}^{-0,799} \quad \text { con } \mathbf{r}^{2}=\mathbf{0 , 7 6 3 4} \\
& \text { Donde: } \\
& \text { Y: Inverso de la velocidad de secado } \\
& \mathrm{X} \text { : Humedad libre promedio } \\
& \text { r: Coeficiente de determinación }
\end{aligned}
$$

Para esto, se ajustó los datos experimentales, para así conseguir la línea de tendencia y la regla de correspondencia; posterior a esto se controló el tiempo de secado (figura 4).

El tiempo de secado fue de 594,36 minutos para la arveja. Se obtuvo a partir de la teoría de secado de los alimentos, considerando tiempos tanto a velocidad constante como decreciente (tabla 2). Dichos tiempos fueron determinados por los métodos analítico y gráfico, haciéndose reportes para cada periodo.

\section{DISCUSIÓN}

En la figura 1, se observa el comportamiento típico de la variación de humedad total en $\mathrm{gH}_{2} \mathrm{O} / \mathrm{g}$ sólidos secos, frente al tiempo transcurrido en minutos; en ella se aprecian los tres períodos de secado antes mencionados correspondiendo el tramo recto al período constante ${ }^{(2)}$. Esta curva es asintótica con el eje horizontal.

En las figuras 2 y 3, se presenta la variación de la velocidad de secado " $\mathrm{R}$ " expresada en $\mathrm{gH}_{2} \mathrm{O} /$ $\mathrm{m}^{2}$.min, frente a la humedad libre promedio visualizándose los tres períodos de secado ${ }^{(3)}$.

De la figura 3 se determinó la velocidad durante el periodo en la que ésta permanece constante, $\mathrm{R}_{\mathrm{c}}=$ o,oolo8 $\mathrm{gH}_{2} \mathrm{O} / \mathrm{m}^{2}$.min ${ }^{(4)}$.

También se determinaron otros parámetros de la cinética de secado, considerados de importancia en el presente trabajo de investigación ${ }^{(5)}$, tales como la Humedad libre crítica $\left(\mathrm{X}_{\mathrm{c}}\right)$, que para la muestra analizada fue de aproximadamente $0,501 \mathrm{gH}_{2} \mathrm{O} / \mathrm{g}$ sólidos secos; la Humedad de equilibrio obtenida 


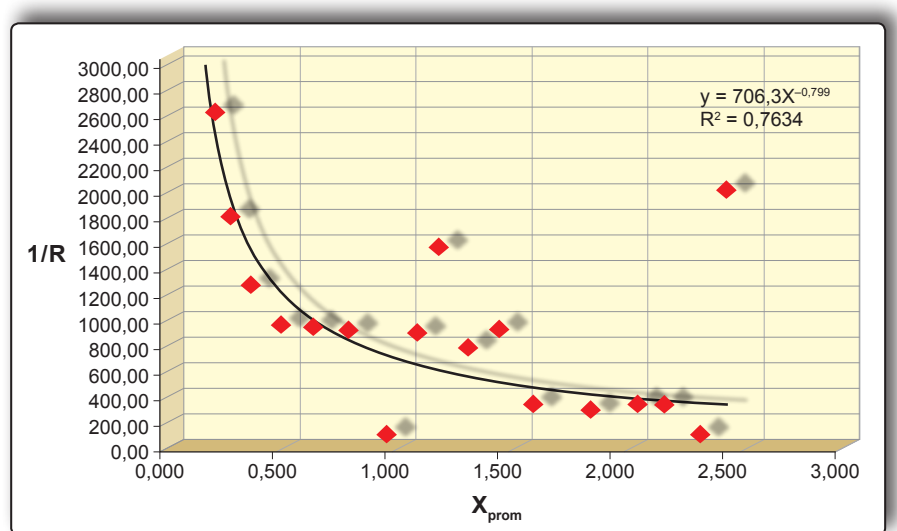

Figura 4. Ajuste de la curva de secado de la arveja: $1 / \mathrm{R}-\mathrm{X}_{\mathrm{prom}}$. 1/R : Inversa de la velocidad de secado en $\mathrm{m}^{2} \cdot \mathrm{min} / \mathrm{gH}_{2} \mathrm{O}$ $\mathrm{X}_{\text {prom }}$ : Humedad libre promedio en $\mathrm{gH}_{2} \mathrm{O} / \mathrm{g}$ sólidos secos

Tabla 1. Secado de arveja.

\begin{tabular}{ccc}
\hline Muestra & $\begin{array}{c}\text { Humedad crítica } \\
\left(\mathbf{g} \mathbf{H}_{2} \mathbf{O} / \mathbf{g} \text { sólidos } \text { secos }\right)\end{array}$ & $\begin{array}{c}\text { Velocidad de secado } \\
\left(\mathbf{g H} \mathbf{O} / \mathbf{m i n}_{\mathbf{m}} \mathbf{)}\right)\end{array}$ \\
\hline $\begin{array}{c}\text { Arveja } \\
\text { deshidratada }\end{array}$ & 0,501 & 0,00108 \\
\hline
\end{tabular}

Tabla 2. Tiempo en los diferentes periodos de secado.

\begin{tabular}{cc}
\hline Periodo de secado & Tiempo (minutos) \\
\hline A velocidad decreciente & 369,0875 \\
A velocidad constante & 225,2715 \\
\hline
\end{tabular}

hasta peso constante durante el secado estimada en o,o71 $\mathrm{g} \mathrm{H}_{2} \mathrm{O} / \mathrm{g}$ sólidos secos.

Al respecto, Lewis ${ }^{(6)}$, señala que este período se caracteriza por estar la superficie del alimento siempre húmeda y a la temperatura de bulbo húmedo del aire, terminando cuando el alimento alcanza su humedad crítica.

En el período de secado a velocidad constante se mantuvo una Tbs (temperatura de bulbo seco) moderadamente elevada de $50^{\circ} \mathrm{C}$, baja humedad relativa y una velocidad media de $3 \mathrm{~m} / \mathrm{s}^{(7)}$.

En las figuras 3 y 4 se presentan las curvas para determinar el tiempo de secado para el período decreciente ${ }^{(2,8)}$. Dado que no se conoce la relación funcional entre la Velocidad de secado (R) y la Humedad libre promedio $\left(\mathrm{X}_{\text {prom }}\right)$, se recurrió a una determinación por regresión potencial para obtener la mejor línea de ajuste, con un valor del coeficiente de determinación $\left(\mathrm{r}^{2}\right)$ de 0,764 y la función de: $\mathrm{Y}=706,3 \mathrm{X}^{-0,799}$; de tal forma presenta un $76 \%$ de correlación entre las variables independiente y dependiente. El resultado es aceptable y confiable porque supera el 70\% de correlación. Con esta función se podría determinar la velocidad de secado y el tiempo de secado, teniendo en cuenta que esta no es la única forma para determinar el tiempo de secado ${ }^{(2,3)}$.
En el período decreciente, la velocidad de transferencia de masa es la que controla el secado; esta agua o vapor de agua que migra hacia la superficie está ligada a los constituyentes de la arveja y su desplazamiento es lento, lo que se corrobora con el tiempo hallado de 6,2 horas ${ }^{(2,3)}$. La velocidad de salida del agua en este período depende de la temperatura del aire y del tamaño de la partícula de alimento, no afectándole la humedad relativa ni la velocidad del aire ${ }^{(9,10)}$.

\section{CONCLUSIONES}

- Los parámetros de la cinética de secado de la arveja verde son: Velocidad de secado $\mathrm{R}_{\mathrm{c}}=$ o,oo108 $\mathrm{gH}_{2} \mathrm{O} / \mathrm{m}^{2}$.min; Tiempo de secado 594,36 minutos, Humedad de equilibrio o,o71 $\mathrm{gH}_{2} \mathrm{O} / \mathrm{g}$ sólidos secos y Humedad crítica o,501 $\mathrm{gH}_{2} \mathrm{O} / \mathrm{g}$ sólidos secos. El tiempo total de secado, sin incluir el período de inducción, fue de 10,0 horas.

- La variedad Usui de la arveja verde presenta una humedad de $72 \%$, sólidos totales de $28 \%$ y, la función de la inversa de velocidad de secado y = $706,3 \mathrm{x}^{-0,799} ; \mathrm{r}^{2}$ es $76,4 \%$.

\section{REFERENCIAS BIBLIOGRÁFICAS}

1. Zhai S, Su H, Taylor R y Slater NKH. Pure ice sublimation within vials in a laboratory lyophiliser; comparison of theory with experiment. Chemical Engineering Science 2005, 6o(4): 1167-76.

2. Geankoplis CJ. Procesos de Transporte y Operaciones Unitarias. $3^{\text {a }}$ ed. Cecsa. México DF, 1998.

3. Fang Y, Selomulya $C$ y Chen XD. On measurement of food powder reconstitution properties. Drying Technology 2007, 26(1): 3-14.

4. Kreith F, Bohn M. Principios de Transferencia de Calor. $1^{\mathrm{a}}$ ed. Thomson Learning. México DF, 2000.

5. Himmelblau DM. Basic Principles and Calculations in Chemical Engineering. 6 $6^{\mathrm{a}}$ ed. Prentice Hall. México DF, 1997.

6. Lewis MJ. Propiedades Físicas de los alimentos y los sistemas de Procesado. $1^{\text {a }}$ ed. Acribia. Zaragoza, 1993.

7. Manrique J. Transferencia de Calor. $2^{\mathrm{a}}$ ed. Oxford University Press. México DF, 2002.

8. vvavSingh $\mathrm{P}$, Heldman D. Introduction to Food Engineering. $4^{\mathrm{a}}$ ed. Elsevier Publishing. London, 2009.

9. Barbosa GV, Vega H. Deshidratación de alimentos. $1^{\mathrm{a}}$ ed. Acribia. Zaragoza, 2000.

\section{Correspondencia}

Nombre: Ing. Javier Saúl Córdova Ramos

Dirección: Jr. Puno 1002 - Lima o1 - Perú

E-mail: javier.cordova.ramos@hotmail.com 